Pacific

Journal of

Mathematics

\title{
SOME COMMENTS ON WEYL'S COMPLETE REDUCIBILITY THEOREM
}

JONATHAN ROGAWSKI AND V. S. VARADARAJAN 


\title{
SOME COMMENTS ON WEYL'S COMPLETE REDUCIBILITY THEOREM
}

\author{
JONATHAN ROGAWSKI AND V. S. VARADARAJAN
}

In memoriam: Jonathan Rogawski

\begin{abstract}
In this note we discuss a purely algebraic proof of Weyl's theorem that all finite-dimensional representations of a complex semisimple Lie algebra are completely reducible. We give a simple and direct proof which is elementary in the sense that it does not use cohomology, and which is a synthesis of the older proofs of Casimir - van der Waerden and of Brauer.
\end{abstract}

\section{Introduction}

In the theory of semisimple Lie algebras, one of the central results is the theorem of Weyl that says that all finite-dimensional representations of a complex semisimple Lie algebra $\mathfrak{g}$ are completely reducible, that is, direct sums of the irreducible ones. In this note we present a purely algebraic proof of this result. Our aim is pedagogical, and so we have made an effort to explain in detail facts that are usually taken for granted in expositions, so as to make this accessible to graduate students and advanced undergraduates. The result is valid over any field of characteristic 0 ; but this generalization can be deduced from the complex case by standard arguments. Hence we restrict ourselves to working over $\mathbb{C}$.

The complete reducibility theorem was first proved by Hermann Weyl [1968] in his great series of papers on the theory of representations of semisimple Lie groups. Although Elie Cartan had already obtained a complete description of the irreducible representations, he did not go seriously into the issue of how an arbitrary representation could be built out of irreducible representations.

Weyl's proof remains one of the most beautiful in the entire theory of representations. The basis of his proof is the following: there is a real form $\mathfrak{u}$ of $\mathfrak{g}$, the so-called compact form, with the property that the simply connected group $U$ corresponding to $\mathfrak{u}$ is compact; here we recall that to say that $\mathfrak{u}$ is a real form of $\mathfrak{g}$ is to require that $\mathfrak{u}$ is a real Lie subalgebra of $\mathfrak{g}$ whose real dimension is the complex dimension of $\mathfrak{g}$.

MSC2010: 17B20, 22E46.

Keywords: Weyl's reducibility theorem. 
Once the existence of $\mathfrak{u}$ is assumed, the proof is straightforward. The representations of $\mathfrak{g}$ correspond naturally to the representations of $\mathfrak{u}$; in one direction one restricts from $\mathfrak{g}$ to $\mathfrak{u}$ and in the other direction one extends from $\mathfrak{u}$ to $\mathfrak{g}$ by complexification. On the other hand, there is a natural correspondence between the representations of $\mathfrak{u}$ and the continuous representations of $U$. Now the compactness of $U$ means that any continuous representation of $U$ is unitarizable, that is, is equivalent to a unitary representation, namely a continuous homomorphism of $U$ into the group of unitary operators on a finite-dimensional complex Hilbert space. So, assuming the representation is unitary, its complete reducibility is immediate, because for any $U$-invariant subspace $M$, its orthogonal complement $M^{\perp}$ is also $U$-invariant and is moreover complementary to $M$; the complete irreducibility is then clear by induction on the dimension of the representation. The unitarizability of any representation of $U$ means that if we start with any representation, we can find a $U$-invariant scalar product in the representation space. To do this, we start with an arbitrary scalar product in the representation space and then introduce the scalar product which is the group average of its transforms by elements of $U$; this latter scalar product is invariant under $U$ so that $U$ is unitary with respect to it (see [Varadarajan 1984, Chapter 4, §4.11]; see also [Hawkins 2000, pp. 465-484]).

This proof is essentially transcendental: it uses invariant integration on the compact group and also the topological fact that the universal covering group of the adjoint group of the compact form is still compact. And neither of these is elementary. The reduction to the compact form $\mathfrak{u}$ and the compact group $U$ was named the unitarian trick by Weyl.

It must be mentioned that the idea of averaging on special compact groups (such as the orthogonal group) goes back to Hurwitz and Schur. For Hurwitz, the goal was to prove the finite generation of the invariants for actions of $\operatorname{SL}(n, \mathbb{C})$ and $\mathrm{SO}(n, \mathbb{C})$. Although these groups are not compact, they contain the compact groups $\mathrm{SU}(n)$ and $\mathrm{SO}(n, \mathbb{R})$, and invariance with respect to these will imply invariance with respect to the complex groups they are contained in (this is the first instance of the unitarian trick); this is because a holomorphic function on the complex groups which vanishes on the relevant compact subgroup is identically 0. For Schur, the goal was the representation theory of the compact groups $\mathrm{SU}(n)$ and $\mathrm{SO}(n, \mathbb{R})$, and he used integration over the group to determine all the irreducible representations, their characters, and their dimensions. The work of Hurwitz and Schur triggered Weyl's imagination, and after he got the results for all semisimple compact groups, he described them in a letter to Schur (see [Borel 1986] for references to the work of Hurwitz and Schur and to Weyl's letter to Schur).

So in the years after Weyl's proof appeared, the question of a purely algebraic proof became a natural issue. The first such algebraic proof was given by Casimir 
and van der Waerden [1935]. ${ }^{1}$ They give a beautiful purely algebraic argument which is elementary in the sense that it relies only on the known structure of irreducible modules as highest-weight modules, and the Casimir operator. A key element of their proof is a delicate calculation in $\mathfrak{s l}(2)$.

After the original proof, additional algebraic proofs appeared in [Brauer 1936] and [Rashevski 1953]. There was also a proof that was cohomological: here it is a question of first establishing that $H^{1}(\mathfrak{g}, M)=0$ for any semisimple Lie algebra $\mathfrak{g}$ and any finite-dimensional $\mathfrak{g}$-module $M$, from which the complete reducibility will follow via standard arguments (see [Varadarajan 1984, Chapter 3, §§3.12-3.13]).

In this note we shall present a proof which is in some sense a synthesis of the earlier proofs of [Casimir and van der Waerden 1935; Brauer 1936]. It is very short and very direct. It uses the Casimir operator in an essential manner (as do all other proofs), the fact that any irreducible module is the one with highest weight equal to some $\lambda$, and the fact that it is enough to lift invariant vectors from the quotient of a module to the module itself. All modules considered from now on are finite-dimensional.

For a detailed historical account of Weyl's theorem and the various proofs of it, see [Borel 1998].

\section{The Casimir operator and the lifting of invariant vectors}

Let $\mathfrak{g}$ be a complex semisimple Lie algebra. If $\left(X_{i}\right)$ is a basis for $\mathfrak{g}$ and $\left(X^{i}\right)$ is the dual basis with respect to the Cartan-Killing form $(\cdot, \cdot)$ (that is, $\left.\left(X_{i}, X^{j}\right)=\delta_{i j}\right)$, then the Casimir operator is

$$
\omega=\sum_{i} X_{i} X^{i}
$$

it is independent of the choice of the basis and lies in the center of the enveloping algebra of $\mathfrak{g}$. It commutes with the action of $\mathfrak{g}$ and hence it goes into a scalar in any irreducible module for $\mathfrak{g}$. We write $g_{\lambda}$ for the value of this scalar when the module is $V_{\lambda}$, the irreducible module of highest weight $\lambda$. Here we have chosen a Cartan subalgebra $\mathfrak{h}$, and a positive system of roots, so that $\lambda \in \mathfrak{h}^{*}$. The isomorphism between $\mathfrak{h}$ and $\mathfrak{h}^{*}$ carries the form on $\mathfrak{h}$ to one on $\mathfrak{h}^{*}$ which is denoted by the same symbol. It is known that $(\cdot, \cdot)$ is real and positive definite on the real span of the roots, which includes all the highest weights. If $\lambda$ is a highest weight, $(\lambda, \alpha) \geq 0$ for all roots $\alpha>0$.

The first lemma is the calculation of $g_{\lambda}$. As usual, let

$$
\rho=\frac{1}{2} \sum_{\alpha>0} \alpha
$$

${ }^{1}$ V. S. V. was fortunate to hear an exposition by Casimir himself of this proof in a conference in Utrecht in 1985. See the Appendix. 
Lemma 1 [Casimir and van der Waerden 1935]. The scalar $g_{\lambda}$ is given by

$$
g_{\lambda}=(\lambda+\rho, \lambda+\rho)-(\rho, \rho) .
$$

Moreover,

$$
g_{\lambda}=0 \Longleftrightarrow \lambda=0 .
$$

In other words, an irreducible module where $\omega$ is 0 is necessarily the trivial module. Proof. We select an ON basis $\left(K_{i}\right)$ for $\mathfrak{h}$ and root vectors $X_{\alpha}$ with $\left(X_{\alpha}, X_{-\alpha}\right)=1$ for all roots $\alpha$. Then

$$
\omega=\sum_{i} K_{i}^{2}+\sum_{\alpha>0}\left(X_{\alpha} X_{-\alpha}+X_{-\alpha} X_{\alpha}\right)=\sum_{i} K_{i}^{2}+\sum_{\alpha>0} H_{\alpha}+2 \sum_{\alpha>0} X_{-\alpha} X_{\alpha} .
$$

If $m_{\lambda}$ is a highest-weight vector for $M_{\lambda}$, then

$$
\omega m_{\lambda}=g_{\lambda} m_{\lambda} .
$$

Since the $X_{\alpha}(\alpha>0)$ annihilate $m_{\lambda}$ and $H m_{\lambda}=\lambda(H) m_{\lambda}$ for $H \in \mathfrak{h}$, it follows that

$$
\begin{aligned}
g_{\lambda} & =\sum_{i} \lambda\left(K_{i}\right)^{2}+\sum_{\alpha>0} \lambda\left(H_{\alpha}\right)=(\lambda, \lambda)+\sum_{\alpha>0}\left(\lambda, H_{\alpha}\right) \\
& =(\lambda, \lambda)+2(\lambda, \rho)=(\lambda+\rho, \lambda+\rho)-(\rho, \rho) .
\end{aligned}
$$

If $\lambda=0$, then $g_{\lambda}=0$. Conversely, suppose that $g_{\lambda}=0$. Then $(\lambda, \lambda)+2(\lambda, \rho)=0$. As both $(\lambda, \lambda)$ and $(\lambda, \rho)$ are $\geq 0$, we must have $(\lambda, \lambda)=0$, so that $\lambda=0$.

The second lemma is that invariant vectors can be lifted. A vector $v$ in a $\mathfrak{g}$-module is invariant if $\mathfrak{g} v=0$.

Lemma 2. Let

$$
M \rightarrow P \rightarrow 0
$$

be an exact sequence of $\mathfrak{g}$-modules and let $p \in P$ be an invariant vector. Then we can lift $p$ to an invariant vector in $M$, namely, find an invariant $u \in M$ such that $u$ maps to $p$.

Proof. Replacing $M$ by the preimage of the line $\mathbb{C} p$, we may assume that $P=\mathbb{C} p$. Now $M$ is the direct sum of the generalized subspaces $M_{r}$ of the Casimir operator, $M_{r}$ being the largest subspace where $\omega$ has the single eigenvalue $r$. The $M_{r}$ are stable under $\mathfrak{g}$, and as $\omega p=0$, it follows that all the $M_{r}$ for $r \neq 0$ map to 0 in $P$. Since $M$ maps onto $P$, this means that $M_{0} \neq 0$ and maps onto $P$. In other words, we may assume that $M=M_{0}$. But then, by Lemma $1, M$ has a Jordan composition series consisting only of trivial modules. Let $H_{i}, X_{i}, Y_{i}(1 \leq i \leq r=\operatorname{rank}$ of $\mathfrak{g})$ be the usual Chevalley generators for $\mathfrak{g}$. Then, for each $i, H_{i}$ has the sole eigenvalue 0 in $M$. If $M\left[H_{i}: a\right]$ is the generalized eigensubspace of $H_{i}$ for the eigenvalue $a$, it is standard that $X_{i}$ (resp. $Y_{i}$ ) maps $M\left[H_{i}: a\right]$ into $M\left[H_{i}: a+2\right]$ (resp. $M\left[H_{i}: a-2\right]$ ). 
But as 0 is the only eigenvalue for $H_{i}$ in $M$, it follows that $X_{i}$ and $Y_{i}$ act as 0 on $M$. But then $H_{i}=\left[X_{i}, Y_{i}\right]$ also acts as 0 , so that $\mathfrak{g}$ acts trivially on $M$. This means that any vector $u$ of $M$ above $p$ satisfies our requirements.

\section{Proof of Weyl's theorem}

Weyl's theorem can now be obtained by a standard general argument.

Weyl's theorem. All $\mathfrak{g}$-modules are completely reducible.

Proof. Suppose $M$ is a $\mathfrak{g}$-module and $N$ a proper submodule. It is a question of finding a submodule $Q$ such that $M=N \oplus Q$. If $P=M / N$, we have an exact sequence of $\mathfrak{g}$-modules

$$
0 \rightarrow N \rightarrow M \rightarrow P \rightarrow 0
$$

So we get the exact sequence $\mathfrak{g}$-modules

$$
0 \rightarrow \operatorname{Hom}(P, N) \rightarrow \operatorname{Hom}(P, M) \rightarrow \operatorname{Hom}(P, P) \rightarrow 0 .
$$

Now the identity $I$ in $\operatorname{Hom}(P, P)$ is an invariant element, and so, by Lemma 2, can be lifted to an invariant element $t$ in $\operatorname{Hom}(P, M)$. Since $t$ maps to $I$, we see that $t$ splits the map $M \rightarrow P$. Since $t$ is invariant, the range $Q$ of $t$ is a submodule of $M$ complementary to $N$.

Remark. The reduction to trivial modules goes back to [Brauer 1936]. It was resurrected by Chevalley [1955] in his proof of Weyl's theorem. Later on, when Mumford [1965] needed a characteristic $p$ version of Lemma 2, he formulated it as follows: if $G$ is a connected semisimple group over an algebraically closed field $K$ of characteristic $p>0$, and $V, W$ are $G$-modules with $V \rightarrow W \rightarrow 0$ exact, and if $w \in W$ is an invariant vector, there are an integer $d>0$ and an invariant vector $v \in S_{d}(V)$ which maps to $w^{d}$ (here $S_{d}$ refers to the component of degree $d$ in the symmetric algebra). It is known that the smallest value of $d$ is a power $p^{m}$ of $p$.

\section{Appendix: A historical note on Casimir and his operator}

Hendrik Brugt Gerhard Casimir (1909-2000) was a physicist whose Leiden thesis [1933] on the theory of diatomic molecules introduced the operator now known as the Casimir operator, as an element of the enveloping algebra of the rotation Lie algebra $\mathfrak{o}$. It commutes with all the elements of $\mathfrak{o}$ and so is a scalar in any irreducible representation of $\mathfrak{o}$. Shortly afterwards, he discovered the analogue of this for any semisimple Lie algebra [1931]. The corresponding operator in any representation of the Lie algebra eventually became known as the Casimir operator. It plays a central role in harmonic analysis and representation theory, even though for groups of higher dimension its value on an irreducible representation no longer determines the representation, unlike what happens for $\mathfrak{s l}(2)$. In [Casimir 1931], he himself pioneered 
the idea that the Casimir operator should be viewed as a second-order differential operator on the group manifold, and that the matrix elements of irreducible representations of the group are eigenfunctions for this operator. The connection between representation theory and differential equations on the group manifold introduced by Casimir found its full force and scope only with the work of Harish-Chandra.

Casimir was one of the great Dutch physicists of the twentieth century who made significant contributions to both experimental and theoretical physics as well as to pure mathematics. In addition, he had a big influence on industry as the head of the research division of Philips. His mathematical contributions include the discovery and use of the Casimir operator as described above. In experimental physics, he predicted what is now known as the Casimir effect, which is a quantum mechanical attraction between conducting plates. His theoretical contributions are quite well known, such as his work on Lars Onsager's microscopic reversibility. One of us (V. S. V.) was present at a conference on semisimple Lie groups in Utrecht in 1985 when Casimir gave a talk on the history of the proof of Weyl's complete reducibility theorem and presented a brief sketch of the algebraic proof given in [Casimir and van der Waerden 1935]. His autobiography [Casimir 1983] is a wonderful document of great interest and humanity.

From the modern perspective, the Casimir operator is an element in the center of the universal enveloping algebra of a semisimple Lie algebra. For simple Lie algebras of dimension greater than 3, the Casimir element does not generate the center of the enveloping algebra. Some of the additional elements of the center were written down by Giulio Racah (1909-1965), an Israeli physicist and mathematician. Racah [1965] determined in some implicit manner the full center of the enveloping algebra of an arbitrary semisimple Lie algebra. The generators of the center discovered by Racah are known to physicists as generalized Casimir operators. For many in the 1950's and early 1960's (including V. S. V.), the Racah notes [1965] were almost the only sources of information on the structure and representations of semisimple Lie algebras till the appearance of [Blanchard et al. 1955] and [Jacobson 1962]. From the mathematical side, the center of the enveloping algebra was first investigated by Harish-Chandra (1923-1983). Harish-Chandra constructed what is now known as the Harish-Chandra isomorphism of the center of the universal enveloping algebra of a general semisimple Lie algebra $\mathfrak{g}$ with the algebra of Weyl group invariants of the algebra of polynomials on a Cartan subalgebra of $\mathfrak{g}$. The center is very closely related to the algebra of polynomial invariants on the Lie algebra, and this was determined by Claude Chevalley (1909-1984), who proved that it is isomorphic, via restriction to a Cartan subalgebra, to the Weyl group invariants of the algebra of polynomials on the Cartan subalgebra. The Harish-Chandra isomorphism, and its $p$-adic twin, the Satake isomorphism, play a fundamental role in harmonic analysis on semisimple groups. 


\section{Acknowledgements}

This paper had its origins in discussions between Jonathan Rogawski and myself. At some point in 2008, although we had a draft in existence, we decided to put it on hold, because of his medical commitments. After Jonathan's untimely passing away in 2011, Don Blasius suggested to me that perhaps this manuscript should see the light of day as an exposition, pedagogical in scope, of a beautiful aspect of semisimple Lie algebras. It is essentially the same as the earlier draft. I thank Don not only for his suggestion to write up these remarks, but also for reading the manuscript and suggesting a number of improvements.

\section{References}

[Blanchard et al. 1955] A. Blanchard, P. Cartier, and M. Lazard, Séminaire "Sophus Lie": Théorie des algèbres de Lie. Topologie des groupes de Lie (Paris, 1954-1955), Secrétariat Mathématique, Paris, 1955. MR 17,384c

[Borel 1986] A. Borel, "Hermann Weyl and Lie groups", pp. 53-82 in Hermann Weyl, 18851985: Centenary lectures (Zürich, 1985), edited by K. Chandrasekharan, Springer, Berlin, 1986. MR 875268 Zbl 0599.01014

[Borel 1998] A. Borel, "Full reducibility and invariants for $\mathrm{SL}_{2}(\mathbb{C})$ ", Enseign. Math. (2) 44:1-2 (1998), 71-90. MR 99h:20001 Zbl 0985.20028

[Brauer 1936] R. Brauer, "Eine Bedingung für vollständige Reduzibilität von Darstellungen gewöhnlicher und infinitesimaler Gruppen", Math. Z. 41:1 (1936), 330-339. Reprinted as pp. 462-471 in his Collected papers, vol. 2, MIT Press, 2003. MR 1545621

[Casimir 1931] H. B. G. Casimir, "Über die Konstruktion einer zu den irreduzibilen Darstellungen halbeinfacher kontinuierlicher Gruppen gehörigen Differentialgleichung”, Proc. Kon. Acad. Amsterdam 84 (1931), 844-846. Zbl 0002.26503

[Casimir 1933] H. B. G. Casimir, Rotation of a rigid body in quantum mechanics, thesis, University of Leiden, 1933. Published by J. H. Voltjers, The Hague. JFM 57.1579.02

[Casimir 1983] H. B. G. Casimir, Haphazard reality: half a century of science, Harper \& Row, New York, 1983.

[Casimir and van der Waerden 1935] H. B. G. Casimir and B. L. van der Waerden, "Algebraischer Beweis der vollständigen Reduzibilität der Darstellungen halbeinfacher Liescher Gruppen”, Math. Ann. 111:1 (1935), 1-12. MR 1512971 Zbl 0011.01101

[Chevalley 1955] C. Chevalley, Théorie des groupes de Lie, III: Théorèmes généraux sur les algèbres de Lie, Actualités Sci. Ind. 1226, Hermann, Paris, 1955. MR 16,901a Zbl 0186.33104

[Hawkins 2000] T. Hawkins, Emergence of the theory of Lie groups: an essay in the history of mathematics, 1869-1926, Springer, New York, 2000. MR 2001f:01031 Zbl 0965.01001

[Jacobson 1962] N. Jacobson, Lie algebras, Interscience Tracts in Pure and Applied Mathematics 10, Interscience, New York, 1962. MR 26 \#1345 Zbl 0121.27504

[Mumford 1965] D. Mumford, Geometric invariant theory, Ergebnisse der Mathematik und ihrer Grenzgebiete, Neue Folge 34, Springer, Berlin, 1965. MR 35 \#5451 Zbl 0147.39304

[Racah 1965] G. Racah, “Group theory and spectroscopy”, Springer Tracts Mod. Phys. 37 (1965), 28-84. MR 35 \#3978 Zbl 0134.43703 
[Rashevski 1953] P. K. Rashevski, "On some fundamental theorems of the theory of Lie groups", Uspekhi Matem. Nauk (N.S.) 8:1(53) (1953), 3-20. In Russian. MR 15,9d Zbl 0050.02504

[Varadarajan 1984] V. S. Varadarajan, Lie groups, Lie algebras, and their representations, Graduate Texts in Mathematics 102, Springer, New York, 1984. MR 85e:22001 Zbl 0955.22500

[Weyl 1968] H. Weyl, Gesammelte Abhandlungen, vol. 2, pp. 543-647, Springer, Berlin, 1968. MR 37 \#6157 Zbl 0164.30103

Received July 16, 2012.

JONATHAN ROGAWSKI

DEPARTMENT OF MATHEMATICS

UNIVERSITY OF CALIFORNIA, LOS ANGELES

LOS ANGELES, CA 90095

UNITED STATES

jonr@math.ucla.edu

V. S. VARADARAJAN

DEPARTMENT OF MATHEMATICS

UNIVERSITY OF CALIFORNIA, LOS ANGELES

Los ANGELES, CA 90095

UNITED STATES 


\title{
PACIFIC JOURNAL OF MATHEMATICS
}

\author{
http://pacificmath.org
}

Founded in 1951 by E. F. Beckenbach (1906-1982) and F. Wolf (1904-1989)

\section{EDITORS}

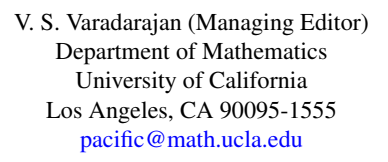

Don Blasius

Department of Mathematics University of California

Los Angeles, CA 90095-1555

blasius@math.ucla.edu

Robert Finn

Department of Mathematics

Stanford University

Stanford, CA 94305-2125

finn@math.stanford.edu

Alexander Merkurjev

Department of Mathematics

University of California

Los Angeles, CA 90095-1555

merkurev@math.ucla.edu

\author{
Vyjayanthi Chari \\ Department of Mathematics \\ University of California \\ Riverside, CA 92521-0135 \\ chari@math.ucr.edu \\ Kefeng Liu \\ Department of Mathematics \\ University of California \\ Los Angeles, CA 90095-1555 \\ liu@math.ucla.edu \\ Sorin Popa \\ Department of Mathematics \\ University of California \\ Los Angeles, CA 90095-1555 \\ popa@math.ucla.edu \\ Paul Yang \\ Department of Mathematics \\ Princeton University \\ Princeton NJ 08544-1000 \\ yang@math.princeton.edu
}

\section{PRODUCTION}

Silvio Levy, Scientific Editor, pacific@math.berkeley.edu

\section{SUPPORTING INSTITUTIONS}

ACADEMIA SINICA, TAIPEI

CALIFORNIA INST. OF TECHNOLOGY

INST. DE MATEMÁTICA PURA E APLICADA

KEIO UNIVERSITY

MATH. SCIENCES RESEARCH INSTITUTE

NEW MEXICO STATE UNIV.

OREGON STATE UNIV.

\author{
STANFORD UNIVERSITY \\ UNIV. OF BRITISH COLUMBIA \\ UNIV. OF CALIFORNIA, BERKELEY \\ UNIV. OF CALIFORNIA, DAVIS \\ UNIV. OF CALIFORNIA, LOS ANGELES \\ UNIV. OF CALIFORNIA, RIVERSIDE \\ UNIV. OF CALIFORNIA, SAN DIEGO \\ UNIV. OF CALIF., SANTA BARBARA
}

\author{
Daryl Cooper \\ Department of Mathematics \\ University of California \\ Santa Barbara, CA 93106-3080 \\ cooper@math.ucsb.edu \\ Jiang-Hua Lu \\ Department of Mathematics \\ The University of Hong Kong \\ Pokfulam Rd., Hong Kong \\ jhlu@maths.hku.hk
}

\section{Jie Qing}

Department of Mathematics

University of California

Santa Cruz, CA 95064

qing@cats.ucsc.edu

These supporting institutions contribute to the cost of publication of this Journal, but they are not owners or publishers and have no responsibility for its contents or policies.

See inside back cover or pacificmath.org for submission instructions.

The subscription price for 2012 is US \$420/year for the electronic version, and \$485/year for print and electronic.

Subscriptions, requests for back issues from the last three years and changes of subscribers address should be sent to Pacific Journal of Mathematics, P.O. Box 4163, Berkeley, CA 94704-0163, U.S.A. Prior back issues are obtainable from Periodicals Service Company, 11 Main Street, Germantown, NY 12526-5635. The Pacific Journal of Mathematics is indexed by Mathematical Reviews, Zentralblatt MATH, PASCAL CNRS Index, Referativnyi Zhurnal, Current Mathematical Publications and the Science Citation Index.

The Pacific Journal of Mathematics (ISSN 0030-8730) at the University of California, c/o Department of Mathematics, 969 Evans Hall, Berkeley, CA 94720-3840, is published monthly except July and August. Periodical rate postage paid at Berkeley, CA 94704, and additional mailing offices. POSTMASTER: send address changes to Pacific Journal of Mathematics, P.O. Box 4163, Berkeley, CA 94704-0163.

PJM peer review and production are managed by EditFlow ${ }^{\circledR}$ from Mathematical Sciences Publishers.

\section{PUBLISHED BY}

\section{mathematical sciences publishers}

http://msp.org/

A NON-PROFIT CORPORATION

Typeset in LATEX 


\section{PACIFIC JOURNAL OF MATHEMATICS}

Volume $260 \quad$ No. $2 \quad$ December 2012

\section{Special issue \\ devoted to the memory of Jonathan Rogawski}

In memoriam: Jonathan Rogawski

257

DON BLASIUS, DINAKAR RAMAKRISHNAN and V. S. VARADARAJAN

$p$-adic Rankin $L$-series and rational points on CM elliptic curves

261

Massimo Bertolini, Henri DARMON and KARTIK PRASANNA

The syntomic regulator for $K_{4}$ of curves

AMNON BESSER and ROB DE JEU

Unique functionals and representations of Hecke algebras

381

BENJAMIN BRUBAKER, DANIEL BUMP and SOLOMON FRIEDBERG

A relative trace formula for PGL(2) in the local setting

395

BROOKE FEIGON

On the degrees of matrix coefficients of intertwining operators

433

TOBIAS FINIS, EREZ LAPID and WERNER MÜLlER

Comparison of compact induction with parabolic induction

457

Guy HENNIART and MARIE-FranCE Vigneras

The functional equation and beyond endoscopy

497

P. EDWARD HERMAN

A correction to Conducteur des Représentations du groupe linéaire

HERVÉ JACQUET

Modular $L$-values of cubic level

ANDREW KNIGHTLY and CHARLES LI

On occult period maps

STEPHEN KUDLA and MiCHAEL RAPOPORT

A prologue to "Functoriality and reciprocity", part I

ROBERT LANGLANDS

Truncation of Eisenstein series

EREZ LAPID and KeITH OUELLETTE

Some comments on Weyl's complete reducibility theorem

JONATHAN ROGAWSKI and V. S. VARADARAJAN

On equality of arithmetic and analytic factors through local Langlands correspondence

FREYDOON SHAHIDI 